\section{INTRAPERITONEAL RUPTURE OF THE BLADDER.}

OPERATION FOUR DAYS AFTER INJURY: RECOVERY. Bx W. PERCY BLUMER, F.R.C.S.EDIN. Surgeon to the Sunderland Infirmary.

J. P. B., labourer, single, aged 35 , was admitted into the Sunderland Infirmary at 1.30 P.M. on August 2nd, 1900 , complaining of pain in the abdomen and inability to pass urine.

History.

On the evening of July $29 \mathrm{ch}$, while walking along a street, he was seized with a feeling of giddiness and fell, striking his abdomen on the kerbstone. On recovery, he felt little the worse for his accident, and walked home. On reaching home he discovered that all attempts at micturition were fruitless; there was, however, a slight escape of what he took to be blood. On July 30 th he stayed in bed and sent for a doctor, who prescribed a pill and saw him only once. He remained in bed till August $2 \mathrm{nd}$, and feeling no better, walked to the infirmary, a distance of over two miles. He had been drinking heavily just before his accident, and said his bladder was full at the time.

\section{Condition on Examination.}

The abdomen was greatly distended all over, somewhat tender on palpation, and absolutely dull in both flanks and across the abdomen to a little above the umbilicus. There was no extravasation into the perineum. Hiccough was constant and distressing; this had been the case for the three days prior to admission. He appeared very ill; the face was pale and drawn, with sunken eyes. No food had been taken, except milk and water, since the accident. Pulse 6o per minute, regular and fairly full. Temperature $96^{\circ} \mathrm{F}$.

A soft rubber catheter passed easily, and immediately bloody urine flowed, 196 ounces in all being drawn out (quantity carefully measured). The abdomen thereafter resumed a more normal appearance. There was no doubt as to the nature of the injury.

Operation.

Laparotomy was performed at 4 P.M., with the assistance of Mr. T. F. Hopgood. The patient having been put under chloroform an incision was made in the middle line just above the pubis, and the abdominal cavity entered in the usual way. A quantity of fluid similar in character to that previously drawn off flowed out. The bladder was lightly contracted, and showed a vertical tear on the posterior wall, which admitted two fingers easily, the peritoneum covering its wall being torn an inch lower down. There was no peritonitis.

The rent was closed with continuous sutures, the bladder tested, the abdominal cavity flushed out with hot boracic solution and the abdominal wound stitched up, a Keith's drainage tube being inserted, and a soft catheter tied into the bladder.

After-History. then again in two hours (fiuid $I$ ounce). Thereafter exhaustion was made every four hours. The tube was withdrawn at the end of forty-eight hours, and the opening closed with a flying suture.

On the evening of August. 5th the patient became very noisy and restless, attempting to get out of bed, pulling out the catheter and threatening the nurses; he suffered also from slight hallucinations. A hypodermic injection of morphine, $\frac{1}{4}$ grain, followed by 1 grain one hour later, had the effect of quieting him, and that night he slept better. On August 6th he was quiet, and slept seven hours.

On August 7 th, when dressing the wound, the lower stitches appeared very tense, and on cutting two a drachm or 80 of pus, and what was evidently urine, escaped. The opening was syringed with $\mathrm{I}$ in 5,000 corrosive solution and drained with iodoform gauze. That evening a consultation was held as to the advisability of opening up the abdominal wound; it was, however, deemed inexpedient on account of the sloughing state of the tissues aud the almost entire absence of symptoms of peritonitis. A fine rubber drainage tube was in. serted instead of the iodoform gauze, and hot boracic fomentations applied every two hours. A seidlitz powder was administered.

There was evidently a little leakage from the bladder, and small quantities of urine continued to flow through the tube in the abdominal wall, the channel being cut off from the general peritoneal cavity.

The remaining stitches were taken out on the tenth day, when the whole length of the wound burst open, and a large slough was taken out. The urine was now very alkaline. On August 9 th, 99 ounces of urine were secreted in the twentyfour hours. The bladder was syringed out with boracic lotion, and boracic acid, 10 grains, was given thrice duily. This treatment was continued for ten days, after which salol was administered in 5-grain doses every two hours, in place of boracic acid, up to 60 grains in the day. Under this treatment the cystitis rapidly yielded.

The temperature was generally 'subnormal for the first ten days, but on the third and tenth days it reached $99^{\circ} \mathrm{F}$. On the sixteenth and twenty-first days it reached $101^{\circ} \mathrm{F}$. It then fell to normal, and remained so until the end.

The fistula was rather slow in healing, but the patient was discharged quite well on October 2gth. - During the last week of his convalescence he had three epileptic fits of a transitory nature.

Remarks.

I have not been able to find on record such a case. The points calling for special mention are :

1. The length of time between the injury and operation.

2. The large amount of fluid in the abdomen causing comparatively little trouble.

3. That four days after the injury the patient walked over two miles.

4. The absence of symptoms of peritonitis.

I am indebted to Dr. O. G. Wotherspoon, house-surgeon, for the notes of this case. His care and attention materially contributed to the patient's recovery.

\section{NOTES ON A CASE OF PROFUSE} HYDROPERITONEUM COMPLICATING UTERINE FIBROIDS.

BY A. C. BUTLER-SMYTHE, F.R.C.S.E.,

Surgeon to the Bamaritan Free Hospital for Women and Children, and to the Grosvenor Hospital for Women and Children, Westminster.

Is abdominal surgery one frequently meets with cases in which uterine fibroids are accompanied by varying quantities of hydroperitoneum, the amount seeming to depend more on the mobility of the growth than on its size. Instances of profuse hydroperitoneum occurring in patients free from thoracic or other abdeminal disease are, however, uncommon, and therefore $I$ have ventured to publish this case.

Mrs. T., aged 40, consulted me in December, 1895, on account of an abdominal swelling

History. - She had been married seventeen years and had had three children, the youngest being then 14 years of age. Menstruation began at 15 , and her periods had always been regular and painless, the los being free and the flow lasting usually seven days. Her last period had occurred in November, 1895 . In her early life she had had the usua minor ailments, and after marriage pneumonia. She had also two attacks of influenza. Six months previous to her visit to me she expe. rienced some abdominal pain, and later on a swelling was noticed in he right side. This swelling seemed to become bigger before each period, and in the intervals it decreased in size. Of late she suffered from con stipation, and there were symptoms of bladder pressure. There had been no loss of flesh within the last six months, her weight being now 8 st. rr lbs. Up to December she had enjoyed good health and was well able to attend to her business and household duties.

Condition when First Seen.-Medium height, pallid features, skin moist, tongue clean, pulse regular and of fair volume, temperature normal, respirations natural, slight hæmic murmur at base, but otherwise the heart seems healthv, bowels constipated. urine free from albunien and sugar, liver normal. Bimanual examination revealed two movable tumours in the abdomen, one situated in the right iliac and lumbar regions, the other occupoing the hypogastric and left inic regions. Pressure on one or both of tho tumours the coun showng the to clo th conner was hara and granulare 32 inches. The diagnosis arrive at was suberions, an it was an absence of severe symptoms. The patient was therefore put upon ergot aiet. 